\section{Protecting against latent TB}

\section{By Michael J. Haas, Senior Writer}

A team of EU and U.S. researchers has created a subunit vaccine based on three Mycobacterium tuberculosis antigens and shown that it protects healthy mice from tuberculosis and prevents reactivation of disease in mouse models of latent infection. ${ }^{1}$ The approach could complement or replace current tuberculosis drug therapies, although there will be challenges in proving the vaccine is safe and effective in latently infected people.

Standard TB prophylaxis relies on the bacillus Calmette-Guérin (BCG) vaccine, which is effective at preventing childhood TB but does not protect against active disease in adults.

Moreover, about $90 \%$ of individuals exposed to M. tuberculosis develop a latent infection that reactivates in $5 \%-10 \%$ of cases if the individual becomes immunocompromised due to age or other conditions such as HIV infection.

Indeed, the World Health Organization estimates that one-third of the world's population is latently infected with TB. Isoniazid, a generic antibiotic, eliminates latent TB in more than $75 \%$ of cases, but compliance is challenging because latently infected individuals do not feel sick and fail to adhere to the months-long drug regimen.

In its previous studies, the EU-U.S. team developed a vaccine, dubbed $\mathrm{H} 1$, based on a dimeric fusion of two bacterial proteins involved in the early stages of TB infection: $M$. tuberculosis secreted antigen 85-B (fbpB; Ag85B) and M. tuberculosis $6 \mathrm{kDa}$ early secretory antigenic target (esxA; Esat6). They found that $\mathrm{H} 1$ protected mice from $\mathrm{TB}$ at least as effectively as BCG. ${ }^{2}$

In its newest study, the team sought to broaden the benefits of $\mathrm{H} 1$ by incorporating into it an antigen involved in latent TB.

The team first conducted gene expression profiling in mouse models and found that an M. tuberculosis protein, M. tuberculosis Rv2660c hypothetical protein (Rv2660c), was expressed at high levels during early and late stages of infection-suggesting the protein played a role in latent infection.

In an unrelated study, a group from the University of Cape Town already had shown the human immune system recognizes Rv2660c. ${ }^{3}$

The two sets of findings supported the team's choice of the protein as a vaccine antigen, team leader Peter Andersen told SciBX.

Next, the team generated a subunit vaccine dubbed H56. It is based on a trimeric Ag85B-Esat6-Rv2660c fusion protein. Inoculation of healthy mice with $\mathrm{H} 56$ and a cationic adjuvant provided greater protection from TB after bacterial challenge than inoculation with
BCG vaccine, the $H 1$ vaccine or vaccines based on Rv2660c, Ag85B or Esat6 alone.

Lastly, in two mouse models of latent TB, H56 and the adjuvant provided greater protection against reactivation of infection than adjuvant alone.

Data were reported in Nature Medicine. Andersen is director of infectious disease immunology and VP of vaccine R\&D at the State Serum Institute (SSI). His team included researchers from the Autonomous University of Barcelona, University College Dublin, Colorado State University and the Stanford University School of Medicine.

"It is exciting that $\mathrm{H} 56$ functions both as a prophylactic and as a therapeutic vaccine," Alexander von Gabain, cofounder and CSO of Intercell AG, told SciBX.

Lewellys Barker, senior medical advisor at Aeras Global TB Vaccine Foundation, agreed. "Given the large fraction of the world's population who are latently infected with tuberculosis and at risk of reactivation, a vaccine to prevent reactivation would be wonderful, and the team's results are encouraging" in this regard, he said.

\section{A therapeutic edge}

Researchers polled by SciBX highlighted some of the potential therapeutic implications of $\mathrm{H} 56$ alone or in combination with other existing approaches.

According to Barker, "the vaccine would have to be used very broadly to substantially reduce the risk and incidence of reactivation" because it is impossible to predict in which latently infected people reactivation will occur. But having to vaccinate only once or twice to prevent reactivation would have tremendous logistical advantages over the existing drug therapy regimens to treat latent $\mathrm{TB}$, he added.

Steven Reed, head of R\&D and founder of the Infectious Disease Research Institute, agreed that a subunit vaccine like H56 is a reasonable strategy for managing latent TB. But he thought that combining H56 and drug therapy could offer better protection to latently infected individuals who become immunocompromised than either approach alone.

"The combination of the vaccine and drugs could reduce the bacterial burden in latently infected individuals so that, if immunosuppression does occur, the risk of reactivation is lower because the number of bacteria is lower," he said.

Furthermore, if reactivation does occur in an immunocompromised individual, Reed said decreasing the bacterial burden also would increase the chances that treatment of the active infection would be successful.

Reed is also founder, president and CEO of Immune Design Corp., which is developing vaccines and vaccine adjuvants to treat cancer and infectious diseases but not TB.

\section{Safety first}

Although researchers agree there is a need for vaccines that improve upon BCG, they said H56 faces major challenges in demonstrating safety and efficacy in latently infected individuals. 
"Safety is a big driver in TB vaccine development, especially for vaccines that will be administered to individuals with latent infection," said Gerald Voss, director of the disease area program for HIV and emerging diseases in GlaxoSmithKline plc's GSK Biologicals unit.

Von Gabain agreed. "In some latently infected people BCG is thought to reactivate the disease," he said.

Demonstrating that a vaccine does not reactivate latent infection has been challenging in preclinical studies because "existing animal models of latent tuberculosis are somewhat artificial" and do not satisfactorily model latency in humans, said Voss.

As an example, he cited the mouse models used by Andersen's team, which were infected with bacteria, treated with antibiotics to suppress the disease and then taken off antibiotics to reactivate the infection.

"Macaques are somewhat more relevant but still have their limitations," Voss added.

Reed countered that a 2009 study ${ }^{4}$ he coauthored with researchers at GSK Biologicals, Colorado State and the Leonard Wood Memorial Center for Leprosy Research demonstrated that "macaques do develop stable, latent infections the way humans do and thus are models of latent tuberculosis."

Nevertheless, he agreed with Voss that "we need to have more data in animal models of latency to have confidence about the safety and efficacy of H56 in humans" with latent TB.

\section{Latent challenges}

Although "latency antigens are a step in the right direction of trying to cover as many stages of tuberculosis disease and the life cycle of the bacteria as possible," Voss also noted that it is still unclear whether targeting an antigen expressed during latent infection-such as Rv2660ccould prevent reactivation of TB in humans.

"Latency is not the same as reactivation, and an antigen that is present in latency may not be present during reactivation," he said. "It would be ideal to see more experiments that elucidated the roles of Rv2660c and other antigens in latency and/or reactivation."

Voss added that H56 would face a clinical hurdle in showing it prevented reactivation because latent infection cannot be unequivocally detected.

For example, the skin test used to detect latent disease could identify trial participants who are TB naïve, "but prior vaccination with BCG can cause a positive reaction to the skin test, making it difficult to distinguish BCG-vaccinated and latently infected people," Voss said.

He noted that the skin test itself elicits an immune response that could interfere with the vaccine.
Thus, Voss cautioned it might not feasible to test for latent TB during H56 trial enrollment. This in turn would make it difficult to determine whether any active disease observed in the participants resulted from the vaccine's failure to protect from primary infection or its failure to prevent reactivation of latent infection.

Andersen said his team is now testing whether H56 can prevent TB in healthy macaques, "with long-term follow-up to observe the late-stage activity of the vaccine."

He agreed it would be interesting to elucidate the precise role Rv2660c plays in latency and/or reactivation, "but it's not something we would do. Other laboratories are better equipped to address this issue."

SSI holds an issued patent covering the use of Rv2660c as a TB vaccine antigen and has applied for a patent covering the use of H56 to prevent TB and reactivation of latent infection. All of that IP is available for licensing or partnering and SSI is seeking corporate partners to develop H56, Petersen said.

In the meantime, sanofi-aventis Group has SSI's H1 vaccine formulated with Intercell's IC31 adjuvant in Phase I testing.

GSK, Aeras and Intercell have several TB vaccines in development (see Table 1 in Taroncher-Oldenburg, G., SciBX 2(9); doi:10.1038/ scibx.2009.345).

Haas, M.J. SciBX 4(6); doi:10.1038/scibx.2011.182

Published online Feb. 17, 2011

REFERENCES

1. Aagaard, C. et al. Nat. Med.; published online Jan. 23, 2011; doi:10.1038/nm.2285

Contact: Claus Aagaard, State Serum Institute, Copenhagen, Denmark e-mail: caa@ssi.dk

2. Weinreich Olsen, A. et al. Infect. Immun. 69, 2773-2778 (2001)

3. Govender, L. et al. Vaccine 29, 51-57 (2010)

4. Reed, S.G. et al. Proc. Natl. Acad. Sci. USA 106, 2301-2306 (2009)

COMPANIES AND INSTITUTIONS MENTIONED

Aeras Global TB Vaccine Foundation, Rockville, Md.

Autonomous University of Barcelona, Badalona, Spain

Colorado State University, Fort Collins, Colo.

GlaxoSmithKline plc (LSE:GSK; NYSE:GSK), London, U.K. Immune Design Corp., Seattle, Wash.

Infectious Disease Research Institute, Seattle, Wash. Intercell AG (VSE:ICLL; OTCQX:INRLY), Vienna, Austria

Leonard Wood Memorial Center for Leprosy Research,

Cebu City, Philippines

sanofi-aventis Group (Euronext:SAN; NYSE:SNY), Paris, France

Stanford University School of Medicine, Stanford, Calif.

State Serum Institute, Copenhagen, Denmark

University of Cape Town, Cape Town, South Africa

University College Dublin, Dublin, Ireland

World Health Organization, Geneva, Switzerland 